\title{
Tests of equivalence and non-inferiority for diagnostic accuracy based on the paired areas under ROC curves
}

\author{
Jen-Pei Liu ${ }^{1,2, *, \dagger}$, Mi-Chia $\mathrm{Ma}^{3, \ddagger}$, Chin-yu $\mathrm{Wu}^{3, \S}$ and Jia-Yen Tai ${ }^{1, \uparrow}$ \\ ${ }^{1}$ Division of Biometry, Department of Agronomy, National Taiwan University, Taipei, Taiwan \\ ${ }^{2}$ Division of Biostatistics and Bioinformatics, National Health Research Institutes, Zhunan, Taiwan \\ ${ }^{3}$ Department of Statistics, National Cheng-kung University, Tainan, Taiwan
}

\begin{abstract}
SUMMARY
Assessment of equivalence or non-inferiority in accuracy between two diagnostic procedures often involves comparisons of paired areas under the receiver operating characteristic (ROC) curves. With some pre-specified clinically meaningful limits, the current approach to evaluating equivalence is to perform the two one-sided tests (TOST) based on the difference in paired areas under ROC curves estimated by the non-parametric method. We propose to use the standardized difference for assessing equivalence or non-inferiority in diagnostic accuracy based on paired areas under ROC curves between two diagnostic procedures. The bootstrap technique is also suggested for both non-parametric method and the standardized difference approach. A simulation study was conducted empirically to investigate the size and power of the four methods for various combinations of distributions, data types, sample sizes, and different correlations. Simulation results demonstrate that the bootstrap procedure of the standardized difference approach not only can adequately control the type I error rate at the nominal level but also provides equivalent power under both symmetrical and skewed distributions. A numerical example using published data illustrates the proposed methods. Copyright (C) 2005 John Wiley \& Sons, Ltd.
\end{abstract}

KEY WORDS: paired areas under ROC curves; non-parametric method; standardized difference; two one-sided tests

\footnotetext{
*Correspondence to: Jen-Pei Liu, Division of Biometry, Department of Agronomy, National Taiwan University, 1, Section 4, Roosevelt Road, Taipei, Taiwan.

†E-mail: jpliu@ntu.edu.tw

‡E-mail: mcma@ibm.stat.ncku.edu.tw

E-mail: cywu@ibm.stat.ncku.edu.tw

TE-mail: r93621209@ntu.edu.tw

Contract/grant sponsor: Taiwan National Science Grant; contract/grant numbers: NSC 92-2118-M-006-001, NSC 93-2118-M-006-002
}

Copyright (c) 2005 John Wiley \& Sons, Ltd. 


\section{INTRODUCTION}

For a new diagnostic procedure that is less invasive, less expensive or easier to administer, it is extremely critical to determine whether its diagnostic accuracy is equivalent or noninferior to the current standard procedure. As a result, if the objective of diagnostic trials is to demonstrate that the diagnostic accuracy of the new diagnostic procedure is within a prespecified margin of the current standard procedure, it is referred to as equivalence trials. On the other hand, if the purpose is to show that the diagnostic accuracy of the new procedure is not worse than that of an existing diagnostic procedure within some pre-determined limit, it is then referred to as non-inferiority trials or one-sided equivalence studies. To reduce the variability between subjects, similar to bioequivalence studies [1], a matched-pair design is often employed to evaluate the new and the standard diagnostic procedures in the same subjects. It follows that the endpoints for assessing diagnostic accuracy are correlated. Current statistical methods for evaluation of equivalence or non-inferiority are based on paired binary endpoints such as sensitivity, specificity or proportion of correct diagnosis. These methods include those proposed by Liu et al. [2], Hsueh et al. [3] for the difference in proportions of correct diagnosis or that proposed by Tang et al. [4] for the ratio of proportions of correct diagnosis.

However, sensitivity, specificity, and proportion of correct diagnosis depend upon some specified decision thresholds and cannot provide an overall characterization of the accuracy for the diagnostic procedure. On the other hand, the receiver operating characteristic (ROC) curve is a summary measure for the accuracy of diagnostic procedures. An ROC curve is a plot of the sensitivity (or true positive rate) on the $y$-axis versus its false positive rate (1-specificity) on the $x$-axis in the unit square. The curve is constructed by changing the decision thresholds that define positive and negative test results. Therefore, a ROC curve incorporates both of sensitivity and specificity and accounts for the inherent trade-offs between them as the decision thresholds change [5]. Define $X$ and $Y$ as the random variable of the measurements for the diseased patients and non-diseased subjects, respectively. Therefore, the ROC curve area can be formulated as

$$
\theta=P(X>Y)
$$

It follows that the ROC curve area is the probability that a randomly selected diseased patient has a test result indicating greater suspicion than that of a randomly chosen normal subject [6]. Obuchowski [7] and Zhou et al. [8] applied the two one-sided tests (TOST) to evaluate the two-sided equivalence of two diagnostic procedures based on the non-parametric estimates of the paired ROC curve areas proposed by Delong et al. [9]. However, its performance in terms of size and power was not thoroughly investigated. On the other hand, one might accept a new diagnostic procedure if it can provide a diagnostic accuracy no worse than the standard but at the same time it is safer, easier to administer or costs less. Therefore, the one-sided non-inferiority hypothesis is more relevant in assessment of equivalence between diagnostic procedures. Since the ROC curve area is a measure for separation of the distribution of the diseased patients from that of the non-diseased subjects, therefore, we propose to use the standardized difference for evaluation of equivalence and non-inferiority between diagnostic procedures. In addition, a simulation study was conducted to empirically investigate and compare the size and power of the non-parametric and proposed methods and their respective bootstrap versions. In Section 2, the non-parametric method for evaluation of the 
equivalence and non-inferiority hypotheses is reviewed. The standardized difference approach is given in Section 3. In addition, a bootstrap method is also suggested for the non-parametric method and standardized difference approach in this section. Simulation results are presented in Section 4. In Section 5, a numerical example using a published data set illustrates the proposed method. Discussion and final remarks are provided in Section 6.

\section{EQUIVALENCE AND NON-INFERIORITY HYPOTHESES}

Let $\theta_{1}$ and $\theta_{2}$ be the paired ROC curve areas for the new and the standard diagnostic tests, respectively. The hypothesis for testing equivalence based on the ROC curve areas between the two diagnostic procedures is given as

$$
H_{0}: \theta_{1}-\theta_{2} \geqslant \delta_{U} \quad \text { or } \quad \theta_{1}-\theta_{2} \leqslant \delta_{L} \quad \text { versus } H_{1}: \delta_{L}<\theta_{1}-\theta_{2}<\delta_{U}
$$

where $\delta_{L}<\delta_{U}$ are some pre-determined clinically meaningful equivalence limits.

This hypothesis can be further decomposed into two one-sided hypotheses:

$$
H_{0 l}: \theta_{1}-\theta_{2} \leqslant-\delta_{L} \text { versus } H_{1 l}: \theta_{1}-\theta_{2}>-\delta_{L}
$$

and

$$
H_{0 u}: \theta_{1}-\theta_{2} \geqslant \delta_{U} \quad \text { versus } H_{1 u}: \theta_{1}-\theta_{2}<\delta_{U}
$$

Because the one-sided hypothesis (3) is to verify the ROC curve area of the new diagnostic test is not smaller than that of the standard test within a pre-specified limit, it is referred to as the non-inferiority hypothesis. Similarly, the one-sided hypothesis (4) is referred to as the non-superiority hypothesis.

Suppose a sample of $N\left(N=N_{A}+N_{N}\right)$ individuals undergo a new and the standard diagnostic procedures for predicting a disease and that the test results are based on continuous measurements. We follow the convention that for both tests, higher values of the results are assumed to be associated with the disease of interest. Also suppose that $N_{A}$ of these individuals truly have the disease and the other $N_{N}\left(=N-N_{A}\right)$ individuals do not have the disease. Denote $X_{h i}$ as the values of the measurements of diagnostic test $h$ from the diseased patients, $i=1, \ldots, N_{A} ; h=1$ (new), 2(standard). $Y_{h j}$ are similarly defined for the non-diseased subjects, $j=1, \ldots, N_{N} ; h=1$ (new), 2(standard).

A non-parametric consistent estimate of the ROC curve area for a diagnostic procedure based on Mann-Whitney $U$ statistic is given as $[9,10]$

$$
\hat{\theta}_{h}=\frac{1}{N_{N} N_{A}} \sum_{j=1}^{N_{N}} \sum_{i=1}^{N_{A}} \psi\left(X_{h i}, Y_{h j}\right)
$$

where

$$
\psi(X, Y)= \begin{cases}1 & X>Y \\ \frac{1}{2} & X=Y \\ 0 & X<Y\end{cases}
$$


An asymptotic estimated variance of $\hat{\theta}_{1}-\hat{\theta}_{2}$ is given as $[8,9]$

$$
\operatorname{vâr}\left(\hat{\theta}_{1}-\hat{\theta}_{2}\right)=\frac{1}{N_{A}}\left[s_{10}^{1,1}+s_{10}^{2,2}-2 s_{10}^{1,2}\right]+\frac{1}{N_{N}}\left[s_{01}^{1,1}+s_{01}^{2,2}-2 s_{01}^{1,2}\right]
$$

where

$$
\begin{aligned}
s_{10}^{h, h^{\prime}} & =\frac{1}{N_{A}-1} \sum_{i=1}^{N_{A}}\left[V_{10}^{h}\left(X_{i}\right)-\hat{\theta}_{h}\right]\left[V_{10}^{h^{\prime}}\left(X_{i}\right)-\hat{\theta}_{h^{\prime}}\right] ; \quad h, h^{\prime}=1,2 \\
s_{01}^{h, h^{\prime}} & =\frac{1}{N_{N}-1} \sum_{j=1}^{N_{N}}\left[V_{01}^{h}\left(Y_{j}\right)-\hat{\theta}_{h}\right]\left[V_{01}^{h^{\prime}}\left(Y_{j}\right)-\hat{\theta}_{h^{\prime}}\right] ; \quad h, h^{\prime}=1,2 \\
V_{10}^{h}\left(X_{i}\right) & =\frac{1}{N_{N}} \sum_{j=1}^{N_{N}} \psi\left(X_{h i}, Y_{h j}\right) ; \quad i=1, \ldots, N_{A}, \quad h=1,2
\end{aligned}
$$

and

$$
V_{01}^{h}\left(Y_{j}\right)=\frac{1}{N_{A}} \sum_{i=1}^{N_{A}} \psi\left(X_{h i}, Y_{h j}\right) ; \quad j=1, \ldots, N_{N}, \quad h=1,2
$$

Zhou et al. [8] suggested that the non-inferiority of the new diagnostic procedure is concluded at the $\alpha$ significance level if

$$
Z_{l}=\frac{\hat{\theta}_{1}-\hat{\theta}_{2}-\delta_{L}}{\sqrt{\operatorname{vâr}\left(\hat{\theta}_{1}-\hat{\theta}_{2}\right)}} \geqslant z_{\alpha}
$$

where $z_{\alpha}$ is the upper $100 \alpha$ percentile of the standard normal distribution.

Similarly, the non-superiority hypothesis (4) is rejected if

$$
Z_{u}=\frac{\hat{\theta}_{1}-\hat{\theta}_{2}-\delta_{U}}{\sqrt{\operatorname{vâr}\left(\hat{\theta}_{1}-\hat{\theta}_{2}\right)}} \leqslant-z_{\alpha}
$$

It follows that by the intersection-union principle [11], the two-sided equivalence between the new and standard diagnostic procedures is concluded at the $\alpha$ significant level by the TOST if both non-inferiority and non-superiority hypotheses are rejected at the $\alpha$ significance level.

\section{THE STANDARDIZED DIFFERENCE APPROACH}

As shown in (1), the ROC curve area depends upon the ability of a diagnostic procedure to separate the distribution of the measurements of the diseased patients from that of the non-disease subjects. Therefore, the ROC curve area is a function of the distance in location between the distributions of $X$ and $Y$. To take into consideration the variation of $X$ and $Y$, a measure for the distance in location between the distributions of $X$ and $Y$ is the standardized 
difference defined as below:

$$
\lambda=\frac{\mu_{A}-\mu_{N}}{\sqrt{\sigma_{A}^{2}+\sigma_{N}^{2}}}
$$

where $\mu_{A}, \mu_{N}$ and $\sigma_{A}^{2}, \sigma_{N}^{2}$ are the means and variances of the distributions of the measurements from the diseased patients and the non-diseased subjects, respectively. Theoretically, the value of the ROC curve area is between 0.5 and 1 and the possible range of the standardized difference can be from $-\infty$ to $\infty$. However, in practice, for some aberrant diagnostic procedure, an observed ROC curve area smaller than 0.5 is possible. On the other hand, for most of diagnostic tests, the standardized differences are some finite quantities. Therefore, assessment of equivalence and non-inferiority in the ROC curve areas between two diagnostic procedures could be based on their corresponding standardized differences. In other words, if two diagnostic procedures have equivalent ROC curve areas, then the difference of their standardized differences should be close and is within some clinically meaningful limits.

Let $\mathbf{X}_{i}=\left(X_{1 i}, X_{2 i}\right)^{\prime}$ be a $2 \times 1$ vector for the results of the new and standard diagnostic procedures of diseased patient $i$ from the new and standard diagnostic tests, $i=1, \ldots, N_{A}$. We also assume that $\mathbf{X}_{i}$ follows a bivariate normal distribution with mean vector $\left(\mu_{A 1}, \mu_{A 2}\right)^{\prime}$ and covariance matrix $\Sigma_{A}$ with elements $\sigma_{A 1}^{2}, \sigma_{A 2}^{2}, \rho_{A} \sigma_{A 1} \sigma_{A 2}$, where $\rho_{A}$ is the correlation between measurements of the new and standard diagnostic tests in the diseased patients. Similarly, denote $\mathbf{Y}_{j}=\left(Y_{1 j}, Y_{2 j}\right)^{\prime}$ as the $2 \times 1$ vector for the results of the new and standard diagnostic tests for non-diseased subject $j$ with mean vector $\left(\mu_{N 1}, \mu_{N 2}\right)^{\prime}$ and covariance matrix $\Sigma_{N}$ with elements $\sigma_{N 1}^{2}, \sigma_{N 2}^{2}, \rho_{N} \sigma_{N 1} \sigma_{N 2}$, where $\rho_{N}$ is the correlation between measurements of the new and standard diagnostic tests in the non-diseased subjects, $j=1, \ldots, N_{N}$. In addition, $\mathbf{X}_{i}$ and $\mathbf{Y}_{j}$ are assumed to be mutually independent. It follows that the standardized difference for diagnostic procedure, $h$, is defined as

$$
\lambda_{h}=\left(\mu_{A h}-\mu_{N h}\right) / \sqrt{\sigma_{A h}^{2}+\sigma_{N h}^{2}}, \quad h=1,2
$$

Under normal assumption, it follows that

$$
\begin{aligned}
\theta_{h} & =P\left(X_{h}>Y_{h}\right) \\
& =P\left(X_{h}-Y_{h}>0\right) \\
& =P\left\{\frac{X_{h}-Y_{h}-\left(\mu_{A h}-\mu_{N h}\right)}{\sqrt{\sigma_{A h}^{2}+\sigma_{N h}^{2}}}>\frac{-\left(\mu_{A_{h}}-\mu_{N h}\right)}{\sqrt{\sigma_{A h}^{2}+\sigma_{N h}^{2}}}\right\}
\end{aligned}
$$

Since $X_{h}-Y_{h}$ follows a normal distribution, it follows that

$$
\theta_{h}=\Phi\left(Z>-\frac{\mu_{A h}-\mu_{N h}}{\sqrt{\sigma_{A h}^{2}+\sigma_{N h}^{2}}}\right)
$$




$$
\begin{aligned}
& =\Phi\left(Z<\frac{\mu_{A h}-\mu_{N h}}{\sqrt{\sigma_{A h}^{2}+\sigma_{N h}^{2}}}\right) \\
& =\Phi\left(Z<\lambda_{h}\right)
\end{aligned}
$$

and $\lambda_{h}=\Phi^{-1}\left(\theta_{h}\right), h=1,2$, where $\Phi($.$) is the cumulative standard normal distribution.$

The relationship between $\theta_{h}$ and $\lambda_{h}$ is well known for the normal distribution, see Hauck et al. [12] and Reiser and Guttman [13]. As a result, the difference in the ROC curve areas between the new and standard diagnostic procedures can be transformed into the difference in standardized differences as

$$
\lambda_{1}-\lambda_{2}=\Phi^{-1}\left(\theta_{1}\right)-\Phi^{-1}\left(\theta_{2}\right)
$$

The above relationship between $\lambda_{h}$ and $\theta_{h}$ in (6) is true for a location-scale family of distributions of the difference. The two-sided equivalence hypothesis based on the standardized difference can therefore be formulated as follows:

$$
H_{0}: \lambda_{1}-\lambda_{2} \geqslant \varepsilon_{U} \quad \text { or } \quad \lambda_{1}-\lambda_{2} \leqslant \varepsilon_{L} \quad \text { versus } H_{1}: \varepsilon_{L}<\lambda_{1}-\lambda_{2}<\varepsilon_{U}
$$

where $\varepsilon_{L}<\varepsilon_{U}$ are some pre-determined clinically meaningful equivalence limits which can be determined from $\delta_{L}$ and $\delta_{U}$ by the relationship $\varepsilon_{L}=\left[\Phi^{-1}\left(\theta_{2}+\delta_{L}\right)-\Phi^{-1}\left(\theta_{2}\right)\right]$ and $\varepsilon_{U}=\left[\Phi^{-1}\left(\theta_{2}+\delta_{U}\right)-\Phi^{-1}\left(\theta_{2}\right)\right]$.

Similarly, the one-sided non-inferiority hypothesis based on the standardized difference is given as

$$
H_{0 l}: \lambda_{1}-\lambda_{2} \leqslant \varepsilon_{L} \text { versus } H_{1 l}: \lambda_{1}-\lambda_{2}>\varepsilon_{L}
$$

$\lambda_{h}$ can be consistently estimated by replacing the population moments by their corresponding sample moments as

$$
\hat{\lambda}_{h}=\frac{\bar{X}_{h}-\bar{Y}_{h}}{\sqrt{s_{A h}^{2}+s_{N h}^{2}}}, \quad h=1,2
$$

Furthermore, the estimated asymptotic variances and covariance of $\hat{\lambda}_{1}$ and $\hat{\lambda}_{2}$ are given as

$$
\begin{aligned}
\operatorname{vâr}\left(\hat{\lambda}_{1}\right)= & \frac{1}{s_{A 1}^{2}+s_{N 1}^{2}}\left(\frac{s_{A 1}^{2}}{N_{A}}+\frac{s_{N 1}^{2}}{N_{N}}\right)+\frac{\left(\bar{X}_{1}-\bar{Y}_{1}\right)^{2}}{2\left(s_{A 1}^{2}+s_{N 1}^{2}\right)^{3}}\left(\frac{s_{A 1}^{4}}{N_{A}-1}+\frac{s_{N 1}^{4}}{N_{N}-1}\right) \\
\operatorname{vâr}\left(\hat{\lambda}_{2}\right)= & \frac{1}{s_{A 2}^{2}+s_{N 2}^{2}}\left(\frac{s_{A 2}^{2}}{N_{A}}+\frac{s_{N 2}^{2}}{N_{N}}\right)+\frac{\left(\bar{X}_{2}-\bar{Y}_{2}\right)^{2}}{2\left(s_{A 2}^{2}+s_{N 2}^{2}\right)^{3}}\left(\frac{s_{A 2}^{4}}{N_{A}-1}+\frac{s_{N 2}^{4}}{N_{N}-1}\right) \\
\operatorname{côv}\left(\hat{\lambda}_{1}, \hat{\lambda}_{2}\right)= & \frac{1}{\sqrt{\left(s_{A 1}^{2}+s_{N 1}^{2}\right)\left(s_{A 2}^{2}+s_{N 2}^{2}\right)}}\left(\frac{\hat{\rho}_{A} s_{A 1} s_{A 2}}{N_{A}}+\frac{\hat{\rho}_{N} s_{N 1} s_{N 2}}{N_{N}}\right) \\
& +\frac{\left(\bar{X}_{1}-\bar{Y}_{1}\right)\left(\bar{X}_{2}-\bar{Y}_{2}\right)}{2 \sqrt{\left(s_{A 1}^{2}+s_{N 1}^{2}\right)^{3}\left(s_{A 2}^{2}+s_{N 2}^{2}\right)^{3}}}\left[\frac{N_{A} \hat{\rho}_{A}^{2} s_{A 1}^{2} s_{A 2}^{2}}{\left(N_{A}-1\right)^{2}}+\frac{N_{N} \hat{\rho}_{N}^{2} s_{N 1}^{2} s_{N 2}^{2}}{\left(N_{N}-1\right)}\right]
\end{aligned}
$$


where

$$
\begin{aligned}
& \hat{\rho}_{A}=\frac{\sum_{i=1}^{N_{A}}\left(X_{1 i}-\bar{X}_{1}\right)\left(X_{2 i}-\bar{X}_{2}\right)}{\sqrt{\sum_{i=1}^{N_{A}}\left(X_{1 i}-\bar{X}_{1}\right)^{2}} \sqrt{\sum_{i=1}^{N_{A}}\left(X_{2 i}-\bar{X}_{2}\right)^{2}}}, \quad \hat{\rho}_{N}=\frac{\sum_{j=1}^{N_{N}}\left(Y_{1 j}-\bar{Y}_{1}\right)\left(Y_{2 j}-\bar{Y}_{2}\right)}{\sqrt{\sum_{j=1}^{N_{N}}\left(Y_{1 j}-\bar{Y}_{1}\right)^{2}} \sqrt{\sum_{j=1}^{N_{N}}\left(Y_{2 j}-\bar{Y}_{2}\right)^{2}}} \\
& s_{A h}^{2}=\frac{1}{N_{A}-1} \sum_{i=1}^{N_{A}}\left(X_{h i}-\bar{X}_{h}\right)^{2} \quad \text { and } \quad s_{N h}^{2}=\frac{1}{N_{N}-1} \sum_{i=1}^{N_{N}}\left(Y_{h i}-\bar{Y}_{h}\right)^{2}, \quad h=1,2
\end{aligned}
$$

An estimated asymptotic variance of $\hat{\lambda}_{1}-\hat{\lambda}_{2}$ is then given as

$$
\operatorname{vâr}\left(\hat{\lambda}_{1}-\hat{\lambda}_{2}\right)=\operatorname{vâr}\left(\hat{\lambda}_{1}\right)+\operatorname{vâr}\left(\hat{\lambda}_{2}\right)-2 \operatorname{côv}\left(\hat{\lambda}_{1}, \hat{\lambda}_{2}\right)
$$

By Slutsky theorem [14], asymptotically, $\left.\left[\left(\hat{\lambda}_{1}-\hat{\lambda}_{2}\right)-\left(\lambda_{1}-\lambda_{2}\right)\right] / \sqrt{\operatorname{vâr}\left(\hat{\lambda}_{1}-\hat{\lambda}_{2}\right)}\right]$ follows a standard normal distribution.

The non-inferiority of the new diagnostic procedure is concluded at the $\alpha$ significance level if

$$
Z_{l}^{\prime}=\frac{\hat{\lambda}_{1}-\hat{\lambda}_{2}-\varepsilon_{L}}{\sqrt{\operatorname{vâr}\left(\hat{\lambda}_{1}-\hat{\lambda}_{2}\right)}} \geqslant z_{\alpha}
$$

Similarly, the non-superiority hypothesis is rejected at the $\alpha$ significance level if

$$
Z_{u}^{\prime}=\frac{\hat{\lambda}_{1}-\hat{\lambda}_{2}-\varepsilon_{U}}{\sqrt{\operatorname{vâr}\left(\hat{\lambda}_{1}-\hat{\lambda}_{2}\right)}} \leqslant-z_{\alpha}
$$

The equivalence of the new diagnostic procedure to the standard is declared at the $\alpha$ significance level if both non-inferiority and non-superiority hypotheses are rejected at the $\alpha$ significance level.

Both non-parametric method and standardized difference approach are asymptotic procedures. Normal approximation might not be adequate even with a moderately large sample size. Therefore, we suggest using bootstrap technique $[15,16]$ to empirically obtain the sampling distributions of test statistics for the two methods. In addition, TOST is operationally equivalent to the confidence interval approach [1] which also provides a probable range for the parameters of interest, i.e. difference in the ROC curve areas and standardized differences. As a result, the confidence interval approach is employed for the bootstrap method. The following is the outline for the bootstrap procedures applied to the non-parametric method and the standardized difference approach for evaluation of equivalence and non-inferiority of diagnostic accuracy based on the ROC curve areas:

1. Generate B-independent bootstrap samples of size $N_{A}$ by sampling with replacement from bivariate vectors of the observed measurements of the diseased patients. Similarly, generate B-independent bootstrap samples of size $N_{N}$ by sampling with replacement from bivariate vectors of the observed measurements of the non-diseased subjects.

2. Calculate the estimated difference in the areas under ROC $\hat{\theta}_{1}-\hat{\theta}_{2}$ or standardized difference $\hat{\lambda}_{1}^{*}-\hat{\lambda}_{2}^{*}$. 
3. Repeat (1) and (2) for a large number of times, say 2000 times or more.

4. Compute the lower and upper limits of the $(1-2 \alpha) 100$ per cent bootstrap confidence interval as the $\alpha 100$ per cent and the $(1-\alpha) 100$ per cent quantiles of the bootstrap distribution.

5. Equivalence between the new and standard diagnostic tests is concluded at the $\alpha$ significance level if the lower and upper limits of the $(1-2 \alpha) 100$ per cent bootstrap confidence interval is completely contained either within $\left(\delta_{L}, \delta_{U}\right)$ for the ROC curve area or $\left(\varepsilon_{L}, \varepsilon_{U}\right)$ for the standardized difference, Non-inferiority of the new diagnostic test is reached if the lower limit of the $(1-2 \alpha) 100$ per cent bootstrap confidence interval is greater than $\delta_{L}\left(\varepsilon_{L}\right)$.

\section{SIMULATION STUDY}

The design for the simulation study is a $2 \times 2$ factorial design. The two factors considered in the simulation study are method (with levels standardized difference and non-parametric) and confidence interval procedure (with levels asymptotic and bootstrap). FORTRAN 90 and IMSL's STAT/LIBRARY FORTRAN subroutines were used in the simulation study to investigate and compare empirically the size and power of four methods. Symmetrical equivalence limits of \pm 0.1 were chosen throughout the study for the difference in the ROC curve areas between the new and standard diagnostic tests. The equivalence limits for the standardized difference approach were then obtained by the relationship between $\lambda_{h}$ and $\theta_{h}$ given in (6). To investigate the impact of the symmetric and skewed distributions of the measurements, the data were generated from the bivariate normal and bivariate exponential distributions. However, the ordinal data are commonly recorded measurements of diagnostic procedures. As a result, the ordinal data of 5 categories were also generated.

For the normal data, $N_{A}+N_{N}$ vectors were generated from bivariate normal distribution with mean vector $(0,0)$ and covariance matrix of equal variance of 1 and covariance $\rho$. Then the first $N_{N}$ bivariate normal vectors represent the measurements of the two diagnostic tests from the non-diseased subjects with mean vector $(0,0)$ and covariance matrix of equal variance of 1 and covariance $\rho$. The other $N_{A}$ bivariate normal vectors represent the measurements of the two diagnostic tests from the diseased subjects with the same covariance matrix and mean vector $\left(\sqrt{ } 2 \Phi^{-1}\left(\theta_{1}\right), \sqrt{ } 2 \Phi^{-1}\left(\theta_{2}\right)\right)$, where $\theta_{1}$ and $\theta_{2}$ are the desired ROC curve areas for the new and standard diagnostic tests, respectively. For the ordinal data, bivariate normal variates were first generated. The possible range of the normal variates was divided into 5 intervals of equal length. The intervals represent the ordered categories and the score from 1 to 5 was assigned according to the ascending order of the intervals. Ordinal data were then generated and assigned to the score according to the interval into which the normal variates fall. The method proposed by Moran [17] was used to generate correlated exponential data.

Real examples given by Zhou et al. [8], Obuchowski [18], Parker and Delong [19], and Pepe [20], suggest a range of the ROC curve areas from 0.52 to 0.85 . Therefore, for the normal distribution, the ROC curve areas of the standard diagnostic procedure are selected from 0.6 to 0.85 by an increment of 0.05 for the simulation. We assume that the correlations between the measurements of the new and standard diagnostic tests are same for the diseased patients and the non-disease subjects. Three values of $\rho_{A}=\rho_{N}=\rho=0.1,0.5,0.9$ were chosen to study 
the impact of low, moderate, and high correlations on the four methods. The health regulatory agencies of some countries request that for marketing approval of in vitro diagnostic tests, the results of diagnostic accuracy such as sensitivity, specificity and the ROC curve area based on 200 samples from each of 3 medical centres for a total of 600 samples be submitted. Therefore, to investigate the size and power of the proposed methods, four different total samples of $70,150,200$, and 400 with an equal number of the diseased patients and nondiseased subjects are selected. We believe that these combinations of the ROC curve areas, correlations, and sample sizes cover most situations for evaluation of equivalence and noninferiority between the new and standard diagnostic tests. With respect to the exponential distribution and the ordinal data, the combinations of 0.7 and 0.8 for the ROC curve area with correlations of 0.5 and 0.9 and the sample size of 200 are investigated in the simulation. For each of the combinations, 2000 random samples were generated. The number of the bootstrap samples is set to be 2000 . For a 5 per cent nominal significance level, a simulation study with 2000 random samples implies that 95 per cent of empirical sizes evaluated at the equivalence limits will be within 0.04513 and 0.05487 if the proposed methods can adequately control the size at the nominal level of 0.05 .

Table I presents the empirical sizes for the equivalence and non-inferiority hypotheses under normal distribution. From Table I, the empirical sizes of one-sided non-inferiority test are larger than those of the two-sided equivalence test. However, for evaluation of equivalence hypothesis, when the total sample size is 70 , correlation is 0.1 , and the ROC curve area of the standard diagnostic test is smaller than 0.80, the empirical sizes of all methods are either close to 0 or very lower. The same situation is also observed when correlation is 0.5 and the ROC curve area is smaller than 0.70 . Therefore, for evaluation of equivalence, all four methods are extremely conservative when sample size is 70 . On the other hand, for the same combinations, the empirical sizes for evaluation of non-inferiority hypothesis remain quite close to the nominal level of 0.05 .

The empirical sizes greater than 0.05487 are highlighted in bold in Tables I, III and IV. From Table I, the empirical size increases either as correlation between the new and standard diagnostic tests increases or as the ROC curve area of the standard test increases. On the other hand, the empirical sizes of non-parametric method and its bootstrap version in general are larger than those of the standardized difference approach. Furthermore, for both non-parametric method and the standardized difference approach, the empirical sizes of the bootstrap procedure are smaller than those of its asymptotic counterpart. For the non-parametric method, there are a total of 131 out of 288 empirical sizes (45.49 per cent) greater than 0.05487 . The maximum empirical size for the non-parametric method can reach as high as 0.0930 and 0.0760 , respectively for the asymptotic method and bootstrap procedure. However, the maximum empirical size for the standardized difference approach is 0.0670 and 0.0615 , respectively, for the asymptotic method and bootstrap procedure. When the correlation is 0.5 and the ROC curve area of the standard test is above 0.75 , most of empirical sizes of the non-parametric method and its bootstrap version for both equivalence and non-inferiority hypothesis are larger than 0.05487. This indicates that when the correlation between the new and standard diagnostic tests is at least 0.5 and the ROC curve area is large, the non-parametric method is liberal in testing the equivalence and non-inferiority hypotheses. On the other hand, 88.9 per cent of the empirical sizes of combinations provided by the bootstrap version of the standardized difference approach are below 0.05487 and only one empirical size ( 0.7 per cent) is above 0.06 while 32 empirical sizes ( 22.2 per cent) of the bootstrap version of the non-parametric method 
Table I. Empirical sizes of equivalence and non-inferiority testing under normal distribution with equivalence limit of 0.1 based on the ROC curve area.

\begin{tabular}{|c|c|c|c|c|c|c|c|c|c|}
\hline \multirow[b]{2}{*}{ Hypothesis } & \multirow[b]{2}{*}{$\rho$} & \multirow[b]{2}{*}{$N$} & \multicolumn{7}{|c|}{ ROC curve area for the standard diagnostic test } \\
\hline & & & Method & 0.60 & 0.65 & 0.70 & 0.75 & 0.80 & 0.85 \\
\hline \multirow[t]{46}{*}{ Equivalence } & \multirow[t]{16}{*}{0.1} & \multirow[t]{4}{*}{70} & Nonpar & 0.0000 & 0.0000 & 0.0000 & 0.0045 & 0.0280 & 0.0705 \\
\hline & & & BNP & 0.0000 & 0.0000 & 0.0050 & 0.0035 & 0.0290 & 0.0630 \\
\hline & & & SD & 0.0000 & 0.0005 & 0.0000 & 0.0005 & 0.0155 & 0.0520 \\
\hline & & & BSD & 0.0000 & 0.0005 & 0.0000 & 0.0005 & 0.0170 & 0.0445 \\
\hline & & \multirow[t]{4}{*}{150} & Nonpar & 0.0090 & 0.0255 & 0.0390 & 0.0470 & 0.0610 & 0.0615 \\
\hline & & & BNP & 0.0090 & 0.0285 & 0.0405 & 0.0470 & 0.0580 & 0.0590 \\
\hline & & & SD & 0.0135 & 0.0310 & 0.0435 & 0.0500 & 0.0515 & 0.0435 \\
\hline & & & BSD & 0.0115 & 0.0290 & 0.0400 & 0.0495 & 0.0470 & 0.0410 \\
\hline & & \multirow[t]{4}{*}{200} & Nonpar & 0.0320 & 0.0510 & 0.0425 & 0.0500 & 0.0540 & 0.0685 \\
\hline & & & BNP & 0.0325 & 0.0510 & 0.0435 & 0.0485 & 0.0540 & 0.0660 \\
\hline & & & SD & 0.0360 & 0.0535 & 0.0425 & 0.0440 & 0.0510 & 0.0650 \\
\hline & & & BSD & 0.0335 & 0.0490 & 0.0410 & 0.0440 & 0.0480 & 0.0615 \\
\hline & & \multirow[t]{4}{*}{400} & Nonpar & 0.0535 & 0.0460 & 0.0465 & 0.0595 & 0.0605 & 0.0620 \\
\hline & & & BNP & 0.0540 & 0.0470 & 0.0455 & 0.0565 & 0.0605 & 0.0610 \\
\hline & & & SD & 0.0570 & 0.0455 & 0.0480 & 0.0525 & 0.0580 & 0.0470 \\
\hline & & & BSD & 0.0565 & 0.0450 & 0.0450 & 0.0515 & 0.0550 & 0.0440 \\
\hline & \multirow[t]{16}{*}{0.5} & \multirow[t]{4}{*}{70} & Nonpar & 0.0050 & 0.0100 & 0.0190 & 0.0410 & 0.0665 & 0.0820 \\
\hline & & & BNP & 0.0060 & 0.0115 & 0.0195 & 0.0400 & 0.0610 & 0.0710 \\
\hline & & & SD & 0.0050 & 0.0125 & 0.0185 & 0.0355 & 0.0580 & 0.0480 \\
\hline & & & BSD & 0.0055 & 0.0105 & 0.0195 & 0.0370 & 0.0520 & 0.0450 \\
\hline & & \multirow{4}{*}{150} & Nonpar & 0.0410 & 0.0465 & 0.0520 & 0.0600 & 0.0550 & 0.0680 \\
\hline & & & BNP & 0.0425 & 0.0425 & 0.0510 & 0.0585 & 0.0510 & 0.0640 \\
\hline & & & SD & 0.0380 & 0.0530 & 0.0485 & 0.0885 & 0.0475 & 0.0620 \\
\hline & & & BSD & 0.0395 & 0.0510 & 0.0485 & 0.0565 & 0.0465 & 0.0585 \\
\hline & & \multirow[t]{4}{*}{200} & Nonpar & 0.0525 & 0.0485 & 0.0600 & 0.0545 & 0.0570 & 0.0775 \\
\hline & & & BNP & 0.0515 & 0.0480 & 0.0595 & 0.0530 & 0.0540 & 0.0700 \\
\hline & & & SD & 0.0515 & 0.0470 & 0.0575 & 0.0490 & 0.0450 & 0.0555 \\
\hline & & & BSD & 0.0500 & 0.0440 & 0.0560 & 0.0480 & 0.0405 & 0.0510 \\
\hline & & \multirow[t]{4}{*}{400} & Nonpar & 0.0535 & 0.0520 & 0.0515 & 0.0500 & 0.0550 & 0.0610 \\
\hline & & & BNP & 0.0515 & 0.0510 & 0.0500 & 0.0480 & 0.0520 & 0.0575 \\
\hline & & & SD & 0.0470 & 0.0515 & 0.0510 & 0.0480 & 0.0480 & 0.0465 \\
\hline & & & BSD & 0.0445 & 0.0495 & 0.0510 & 0.0450 & 0.0460 & 0.0485 \\
\hline & \multirow[t]{14}{*}{0.9} & \multirow[t]{4}{*}{70} & Nonpar & 0.0490 & 0.0540 & 0.0685 & 0.0760 & 0.0850 & 0.0930 \\
\hline & & & BNP & 0.0455 & 0.0480 & 0.0610 & 0.0605 & 0.0615 & 0.0760 \\
\hline & & & SD & 0.0540 & 0.0520 & 0.0500 & 0.0540 & 0.0630 & 0.0625 \\
\hline & & & BSD & 0.0435 & 0.0433 & 0.0430 & 0.0435 & 0.0445 & 0.0425 \\
\hline & & \multirow[t]{4}{*}{150} & Nonpar & 0.0565 & 0.0580 & 0.0575 & 0.0626 & 0.0625 & 0.0765 \\
\hline & & & BNP & 0.0525 & 0.0520 & 0.0490 & 0.0550 & 0.0535 & 0.0655 \\
\hline & & & SD & 0.0545 & 0.0550 & 0.0530 & 0.0545 & 0.0555 & 0.0545 \\
\hline & & & BSD & 0.0475 & 0.0415 & 0.0450 & 0.0420 & 0.0465 & 0.0425 \\
\hline & & \multirow[t]{4}{*}{200} & Nonpar & 0.0615 & 0.0555 & 0.0600 & 0.0650 & 0.0730 & 0.0625 \\
\hline & & & BNP & 0.0570 & 0.0525 & 0.0560 & 0.0590 & 0.0650 & 0.0590 \\
\hline & & & SD & 0.0620 & 0.0495 & 0.0475 & 0.0600 & 0.0570 & 0.0670 \\
\hline & & & BSD & 0.0565 & 0.0445 & 0.0405 & 0.0450 & 0.0465 & 0.0490 \\
\hline & & \multirow{2}{*}{400} & Nonpar & 0.0455 & 0.0565 & 0.0550 & 0.0605 & 0.0520 & 0.0600 \\
\hline & & & BNP & 0.0445 & 0.0520 & 0.0510 & 0.0565 & 0.0470 & 0.0555 \\
\hline
\end{tabular}


Table I. Continued.

\begin{tabular}{|c|c|c|c|c|c|c|c|c|c|}
\hline \multirow[b]{2}{*}{ Hypothesis } & \multirow[b]{2}{*}{$\rho$} & \multirow[b]{2}{*}{$N$} & \multirow[b]{2}{*}{ Method } & \multicolumn{6}{|c|}{ ROC curve area for the standard diagnostic test } \\
\hline & & & & 0.60 & 0.65 & 0.70 & 0.75 & 0.80 & 0.85 \\
\hline & & & SD & 0.0515 & 0.0420 & 0.0505 & 0.0500 & 0.0495 & 0.0480 \\
\hline & & & BSD & 0.0425 & 0.0370 & 0.0460 & 0.0460 & 0.0435 & 0.0405 \\
\hline \multirow[t]{44}{*}{ Non-inferiority } & \multirow[t]{16}{*}{0.1} & \multirow[t]{4}{*}{70} & Nonpar & 0.0475 & 0.0480 & 0.0510 & 0.0490 & 0.0600 & 0.0745 \\
\hline & & & BNP & 0.0470 & 0.0515 & 0.0515 & 0.0495 & 0.0600 & 0.0670 \\
\hline & & & SD & 0.0440 & 0.0520 & 0.0515 & 0.0480 & 0.0545 & 0.0550 \\
\hline & & & BSD & 0.0430 & 0.0510 & 0.0500 & 0.0445 & 0.0515 & 0.0485 \\
\hline & & \multirow[t]{4}{*}{150} & Nonpar & 0.0485 & 0.0615 & 0.0545 & 0.0545 & 0.0620 & 0.0615 \\
\hline & & & BNP & 0.0485 & 0.0605 & 0.0560 & 0.0545 & 0.0590 & 0.0590 \\
\hline & & & SD & 0.0475 & 0.0620 & 0.0570 & 0.0550 & 0.0525 & 0.0435 \\
\hline & & & BSD & 0.0465 & 0.0595 & 0.0535 & 0.0560 & 0.0485 & 0.0410 \\
\hline & & \multirow[t]{4}{*}{200} & Nonpar & 0.0435 & 0.0600 & 0.0440 & 0.0520 & 0.0545 & 0.0685 \\
\hline & & & BNP & 0.0440 & 0.0590 & 0.0450 & 0.0505 & 0.0545 & 0.0660 \\
\hline & & & SD & 0.0445 & 0.0590 & 0.0450 & 0.0460 & 0.0510 & 0.0650 \\
\hline & & & BSD & 0.0425 & 0.0555 & 0.0430 & 0.0460 & 0.0485 & 0.0615 \\
\hline & & \multirow[t]{4}{*}{400} & Nonpar & 0.0535 & 0.0460 & 0.0465 & 0.0595 & 0.0605 & 0.0620 \\
\hline & & & BNP & 0.0540 & 0.0470 & 0.0455 & 0.0565 & 0.0605 & 0.0610 \\
\hline & & & SD & 0.0570 & 0.0455 & 0.0480 & 0.0525 & 0.0580 & 0.0470 \\
\hline & & & BSD & 0.0565 & 0.0450 & 0.0450 & 0.0515 & 0.0550 & 0.0440 \\
\hline & \multirow{16}{*}{0.5} & \multirow{4}{*}{70} & Nonpar & 0.0520 & 0.0470 & 0.0485 & 0.0650 & 0.0715 & 0.0820 \\
\hline & & & BNP & 0.0530 & 0.0470 & 0.0500 & 0.0640 & 0.0670 & 0.0710 \\
\hline & & & SD & 0.0520 & 0.0485 & 0.0470 & 0.0565 & 0.0595 & 0.0485 \\
\hline & & & BSD & 0.0460 & 0.0460 & 0.0485 & 0.0545 & 0.0580 & 0.0455 \\
\hline & & \multirow[t]{4}{*}{150} & Nonpar & 0.0470 & 0.0485 & 0.0525 & 0.0600 & 0.0550 & 0.0680 \\
\hline & & & BNP & 0.0480 & 0.0455 & 0.0520 & 0.0590 & 0.0510 & 0.0640 \\
\hline & & & SD & 0.0430 & 0.0540 & 0.0495 & 0.0585 & 0.0475 & 0.0620 \\
\hline & & & BSD & 0.0450 & 0.0520 & 0.0455 & 0.0585 & 0.0465 & 0.0585 \\
\hline & & \multirow[t]{4}{*}{200} & Nonpar & 0.0525 & 0.0485 & 0.0605 & 0.0545 & 0.0570 & 0.0775 \\
\hline & & & BNP & 0.0515 & 0.0480 & 0.0600 & 0.0530 & 0.0540 & 0.0700 \\
\hline & & & SD & 0.0515 & 0.0470 & 0.0575 & 0.0490 & 0.0450 & 0.0555 \\
\hline & & & BSD & 0.0500 & 0.0440 & 0.0560 & 0.0480 & 0.0405 & 0.0510 \\
\hline & & \multirow{4}{*}{400} & Nonpar & 0.0535 & 0.0520 & 0.0515 & 0.0500 & 0.0550 & 0.0610 \\
\hline & & & BNP & 0.0515 & 0.0510 & 0.0500 & 0.0480 & 0.0520 & 0.0575 \\
\hline & & & SD & 0.0470 & 0.0515 & 0.0510 & 0.0480 & 0.0480 & 0.0465 \\
\hline & & & BSD & 0.0445 & 0.0495 & 0.0510 & 0.0450 & 0.0460 & 0.0485 \\
\hline & \multirow[t]{12}{*}{0.9} & \multirow[t]{4}{*}{70} & Nonpar & 0.0490 & 0.0540 & 0.0685 & 0.0760 & 0.0800 & 0.0930 \\
\hline & & & BNP & 0.0455 & 0.0480 & 0.0610 & 0.0605 & 0.0615 & 0.0760 \\
\hline & & & SD & 0.0540 & 0.0520 & 0.0500 & 0.0540 & 0.0630 & 0.0625 \\
\hline & & & BSD & 0.0435 & 0.0435 & 0.0430 & 0.0435 & 0.0445 & 0.0425 \\
\hline & & \multirow[t]{4}{*}{150} & Nonpar & 0.0565 & 0.0580 & 0.0575 & 0.0620 & 0.0625 & 0.0765 \\
\hline & & & BNP & 0.0525 & 0.0520 & 0.0490 & 0.0550 & 0.0535 & 0.0655 \\
\hline & & & SD & 0.0545 & 0.0500 & 0.0530 & 0.0545 & 0.0555 & 0.0545 \\
\hline & & & BSD & 0.0475 & 0.0415 & 0.0450 & 0.0420 & 0.0465 & 0.0425 \\
\hline & & \multirow[t]{4}{*}{200} & Nonpar & 0.0615 & 0.0555 & 0.0600 & 0.0650 & 0.0730 & 0.0625 \\
\hline & & & BNP & 0.0570 & 0.0525 & 0.0560 & 0.0590 & 0.0650 & 0.0590 \\
\hline & & & SD & 0.0620 & 0.0495 & 0.0475 & 0.0600 & 0.0570 & 0.0670 \\
\hline & & & BSD & 0.0565 & 0.0445 & 0.0405 & 0.0450 & 0.0465 & 0.0490 \\
\hline
\end{tabular}


Table I. Continued.

\begin{tabular}{|c|c|c|c|c|c|c|c|c|c|}
\hline \multirow[b]{2}{*}{ Hypothesis } & \multirow[b]{2}{*}{$\rho$} & \multirow[b]{2}{*}{$N$} & \multirow[b]{2}{*}{ Method } & \multicolumn{6}{|c|}{ ROC curve area for the standard diagnostic test } \\
\hline & & & & 0.60 & 0.65 & 0.70 & 0.75 & 0.80 & 0.85 \\
\hline & & 400 & Nonpar & 0.0455 & 0.0565 & 0.0550 & 0.0605 & 0.0520 & 0.0600 \\
\hline & & & BNP & 0.0445 & 0.0520 & 0.0510 & 0.0565 & 0.0470 & 0.0555 \\
\hline & & & SD & 0.0515 & 0.0420 & 0.0505 & 0.0500 & 0.0495 & 0.0480 \\
\hline & & & BSD & 0.0425 & 0.0370 & 0.0460 & 0.0460 & 0.0435 & 0.0405 \\
\hline
\end{tabular}

Note: $\rho$ : Common correlation coefficient of the measurements between the new and standard diagnostic test. Nonpar: Non-parametric method. BNP: Bootstrap procedure of the non-parametric method. SD: Standard difference approach. BSD: Bootstrap procedure of the standard difference approach.

are at least 0.06 . Therefore, simulation results demonstrate that the bootstrap version of the standardized difference approach can adequately control the type I error rate at the nominal level.

Table II provides the empirical powers under normal distribution when the difference of the ROC curve areas is 0.05 . From Table II, the empirical powers of one-sided non-inferiority hypothesis are greater than those of the two-sided equivalence hypothesis. In addition, the empirical power increases as either the sample size increases or the correlation between the new and standard diagnostic tests increases. On the other hand, all four methods provide comparable powers for these combinations. When the correlation between the new and standard diagnostic tests is 0.1 , the maximum empirical power of the four methods for equivalence and non-inferiority hypotheses is only about 0.68 provided by a total sample of 400 . On the other hand, sufficient power $(>0.8)$ can be provided with a total sample size of 150 when the correlation between the new and standard diagnostic tests is 0.9 and the ROC curve area of the standard test is at least 0.75 . Figures 1 and 2 present the power curves of the two-sided equivalence and non-inferiority hypotheses, respectively, when the total sample size is 200, the correlation is 0.9 , and the ROC curve area of the standard diagnostic test is 0.7 . For the two-sided equivalence hypothesis, the power curves increase monotonically as the difference in the ROC curve area increases from -0.15 to 0 . They reach the maximum at 0 and then decrease monotonically as the difference in the ROC curve area increases from 0 to 0.15 . The power curves almost overlap each other in most of range from -0.15 to 0 . However, at equivalence limits of \pm 0.1 , the size of the non-parametric method is larger than 0.05 . In addition, the empirical power curve of equivalence hypothesis is symmetrical about 0 . On the other hand, for the one-sided non-inferiority hypothesis, the power curves are monotonic increasing functions of the difference in the ROC curve areas.

Table III presents the empirical sizes at $\delta=0.1$ and empirical powers at $\theta_{1}-\theta_{2}=0.05$ for the exponential distribution when the total sample size is 200. As shown in Table III, the empirical sizes of the asymptotic non-parametric method and its bootstrap version for both equivalence and non-inferiority hypotheses range from 0.1145 to 0.5090 and the empirical sizes of the standardized difference approach range from 0.075 to 0.127 . Therefore, neither the asymptotic and bootstrap procedures of the non-parametric method, nor the standardized difference approach can control the size at the 5 per cent nominal level when the distribution is skewed. On the other hand, the empirical sizes of the bootstrap version of the standardized difference approach are all below 0.05 . Therefore, the bootstrap method of the standardized 
Table II. Empirical powers of equivalence and non-inferiority testing under normal distribution with equivalence limit of 0.05 based on the ROC curve area.

\begin{tabular}{|c|c|c|c|c|c|c|c|c|c|}
\hline \multirow[b]{2}{*}{ Hypothesis } & \multirow[b]{2}{*}{$\rho$} & \multirow[b]{2}{*}{$N$} & \multicolumn{7}{|c|}{ ROC curve area for the standard diagnostic test } \\
\hline & & & Method & 0.60 & 0.65 & 0.70 & 0.75 & 0.80 & 0.85 \\
\hline \multirow[t]{46}{*}{ Equivalence } & \multirow[t]{16}{*}{0.1} & \multirow[t]{4}{*}{70} & Nonpar & 0.0000 & 0.0000 & 0.0005 & 0.0025 & 0.0285 & 0.1195 \\
\hline & & & BNP & 0.0000 & 0.0000 & 0.0010 & 0.0025 & 0.0305 & 0.1160 \\
\hline & & & SD & 0.0000 & 0.0000 & 0.0000 & 0.0000 & 0.0005 & 0.0255 \\
\hline & & & BSD & 0.0000 & 0.0000 & 0.0000 & 0.0000 & 0.0020 & 0.0325 \\
\hline & & \multirow[t]{4}{*}{150} & Nonpar & 0.0160 & 0.0405 & 0.0830 & 0.1510 & 0.2550 & 0.3695 \\
\hline & & & BNP & 0.0155 & 0.0465 & 0.0865 & 0.1505 & 0.2505 & 0.3640 \\
\hline & & & SD & 0.0220 & 0.0425 & 0.0655 & 0.1250 & 0.1990 & 0.2650 \\
\hline & & & BSD & 0.0210 & 0.0415 & 0.0605 & 0.1170 & 0.1860 & 0.2540 \\
\hline & & \multirow[t]{4}{*}{200} & Nonpar & 0.1205 & 0.1575 & 0.1940 & 0.2570 & 0.3425 & 0.4530 \\
\hline & & & BNP & 0.1245 & 0.1560 & 0.1995 & 0.2565 & 0.3440 & 0.4490 \\
\hline & & & SD & 0.1310 & 0.1510 & 0.1890 & 0.2265 & 0.2780 & 0.3315 \\
\hline & & & BSD & 0.1220 & 0.1450 & 0.1850 & 0.2240 & 0.2745 & 0.3250 \\
\hline & & \multirow[t]{4}{*}{400} & Nonpar & 0.3655 & 0.4115 & 0.4550 & 0.4800 & 0.5695 & 0.6780 \\
\hline & & & BNP & 0.3650 & 0.4090 & 0.4520 & 0.4775 & 0.5660 & 0.6790 \\
\hline & & & SD & 0.3615 & 0.3850 & 0.4165 & 0.4285 & 0.4725 & 0.5200 \\
\hline & & & BSD & 0.3605 & 0.3845 & 0.4145 & 0.4220 & 0.4650 & 0.5150 \\
\hline & \multirow[t]{16}{*}{0.5} & \multirow[t]{4}{*}{70} & Nonpar & 0.0055 & 0.0120 & 0.0335 & 0.0785 & 0.1490 & 0.2850 \\
\hline & & & BNP & 0.0080 & 0.0125 & 0.0340 & 0.0815 & 0.1445 & 0.2730 \\
\hline & & & SD & 0.0055 & 0.0165 & 0.0220 & 0.0535 & 0.1015 & 0.1890 \\
\hline & & & BSD & 0.0065 & 0.0120 & 0.0190 & 0.0555 & 0.0880 & 0.1660 \\
\hline & & \multirow[t]{4}{*}{150} & Nonpar & 0.2120 & 0.2655 & 0.2975 & 0.3355 & 0.4245 & 0.4735 \\
\hline & & & BNP & 0.2115 & 0.2670 & 0.2970 & 0.3355 & 0.4190 & 0.4620 \\
\hline & & & SD & 0.2320 & 0.2755 & 0.2765 & 0.3030 & 0.3510 & 0.3660 \\
\hline & & & BSD & 0.2225 & 0.2645 & 0.2655 & 0.2920 & 0.3360 & 0.3580 \\
\hline & & \multirow[t]{4}{*}{200} & Nonpar & 0.3265 & 0.3355 & 0.3870 & 0.4330 & 0.5010 & 0.6045 \\
\hline & & & BNP & 0.3240 & 0.3370 & 0.3845 & 0.4315 & 0.4925 & 0.5945 \\
\hline & & & SD & 0.3280 & 0.3250 & 0.3625 & 0.3795 & 0.4160 & 0.4660 \\
\hline & & & BSD & 0.3190 & 0.3205 & 0.3505 & 0.3685 & 0.4110 & 0.4475 \\
\hline & & \multirow[t]{4}{*}{400} & Nonpar & 0.5575 & 0.5835 & 0.6125 & 0.6540 & 0.7530 & 0.8480 \\
\hline & & & BNP & 0.5565 & 0.5845 & 0.6170 & 0.6525 & 0.7465 & 0.8375 \\
\hline & & & SD & 0.5655 & 0.5620 & 0.5690 & 0.5965 & 0.6480 & 0.7130 \\
\hline & & & BSD & 0.5645 & 0.5530 & 0.5630 & 0.5890 & 0.6395 & 0.7010 \\
\hline & \multirow[t]{14}{*}{0.9} & \multirow[t]{4}{*}{70} & Nonpar & 0.4470 & 0.4765 & 0.4985 & 0.5385 & 0.5830 & 0.6475 \\
\hline & & & BNP & 0.4185 & 0.4505 & 0.4710 & 0.5120 & 0.5465 & 0.6045 \\
\hline & & & SD & 0.4905 & 0.5050 & 0.5145 & 0.5500 & 0.5610 & 0.6095 \\
\hline & & & BSD & 0.4500 & 0.4685 & 0.4755 & 0.5095 & 0.5140 & 0.5420 \\
\hline & & \multirow[t]{4}{*}{150} & Nonpar & 0.7050 & 0.7510 & 0.7785 & 0.8100 & 0.8450 & 0.9090 \\
\hline & & & BNP & 0.6900 & 0.7340 & 0.7630 & 0.8000 & 0.8335 & 0.8930 \\
\hline & & & SD & 0.7500 & 0.7740 & 0.7935 & 0.8035 & 0.8340 & 0.8800 \\
\hline & & & BSD & 0.7230 & 0.7400 & 0.7735 & 0.7840 & 0.8115 & 0.8550 \\
\hline & & \multirow[t]{4}{*}{200} & Nonpar & 0.8255 & 0.8490 & 0.8705 & 0.8910 & 0.9245 & 0.9590 \\
\hline & & & BNP & 0.8170 & 0.8410 & 0.8615 & 0.8795 & 0.9175 & 0.9530 \\
\hline & & & SD & 0.8570 & 0.8565 & 0.8740 & 0.8790 & 0.9175 & 0.9385 \\
\hline & & & BSD & 0.8440 & 0.8380 & 0.8615 & 0.8665 & 0.9035 & 0.9260 \\
\hline & & \multirow[t]{2}{*}{400} & Nonpar & 0.9795 & 0.9850 & 0.9890 & 0.9930 & 0.9985 & 0.9980 \\
\hline & & & BNP & 0.9770 & 0.9840 & 0.9895 & 0.9925 & 0.9985 & 0.9980 \\
\hline
\end{tabular}


Table II. Continued.

\begin{tabular}{|c|c|c|c|c|c|c|c|c|c|}
\hline \multirow[b]{2}{*}{ Hypothesis } & \multirow[b]{2}{*}{$\rho$} & \multirow[b]{2}{*}{$N$} & \multirow[b]{2}{*}{ Method } & \multicolumn{6}{|c|}{ ROC curve area for the standard diagnostic test } \\
\hline & & & & 0.60 & 0.65 & 0.70 & 0.75 & 0.80 & 0.85 \\
\hline & & & SD & 0.9885 & 0.9920 & 0.9895 & 0.9915 & 0.9965 & 0.9960 \\
\hline & & & BSD & 0.9860 & 0.9890 & 0.9880 & 0.9915 & 0.9960 & 0.9950 \\
\hline \multirow{44}{*}{ Non-inferiority } & \multirow{16}{*}{0.1} & \multirow[t]{4}{*}{70} & Nonpar & 0.1245 & 0.1425 & 0.1455 & 0.1415 & 0.1585 & 0.1745 \\
\hline & & & BNP & 0.1230 & 0.1395 & 0.1395 & 0.1425 & 0.1595 & 0.1730 \\
\hline & & & SD & 0.1165 & 0.1425 & 0.1505 & 0.1560 & 0.2010 & 0.2370 \\
\hline & & & BSD & 0.1205 & 0.1435 & 0.1530 & 0.1635 & 0.2035 & 0.2355 \\
\hline & & \multirow[t]{4}{*}{150} & Nonpar & 0.2080 & 0.1965 & 0.2200 & 0.2305 & 0.2600 & 0.2870 \\
\hline & & & BNP & 0.2045 & 0.1955 & 0.2190 & 0.2210 & 0.2515 & 0.2780 \\
\hline & & & SD & 0.2050 & 0.2040 & 0.2350 & 0.2490 & 0.3055 & 0.3825 \\
\hline & & & BSD & 0.2045 & 0.2080 & 0.2365 & 0.2485 & 0.3010 & 0.3780 \\
\hline & & \multirow[t]{4}{*}{200} & Nonpar & 0.2360 & 0.2535 & 0.2760 & 0.3045 & 0.3525 & 0.4560 \\
\hline & & & BNP & 0.2360 & 0.2510 & 0.2790 & 0.3030 & 0.3535 & 0.4520 \\
\hline & & & SD & 0.2330 & 0.2500 & 0.2670 & 0.2815 & 0.2930 & 0.3350 \\
\hline & & & BSD & 0.2285 & 0.2445 & 0.2650 & 0.2800 & 0.2910 & 0.3290 \\
\hline & & \multirow[t]{4}{*}{400} & Nonpar & 0.3690 & 0.4165 & 0.4580 & 0.4810 & 0.5700 & 0.6780 \\
\hline & & & BNP & 0.3685 & 0.4150 & 0.4555 & 0.4785 & 0.5665 & 0.6790 \\
\hline & & & SD & 0.3640 & 0.3920 & 0.4195 & 0.4295 & 0.4730 & 0.5200 \\
\hline & & & BSD & 0.3630 & 0.3930 & 0.4175 & 0.4230 & 0.4655 & 0.5150 \\
\hline & \multirow[t]{16}{*}{0.5} & \multirow{4}{*}{70} & Nonpar & 0.1845 & 0.1930 & 0.1815 & 0.2095 & 0.2055 & 0.2490 \\
\hline & & & BNP & 0.1770 & 0.1870 & 0.1765 & 0.2075 & 0.1955 & 0.2335 \\
\hline & & & SD & 0.1720 & 0.1960 & 0.1970 & 0.2395 & 0.2450 & 0.3340 \\
\hline & & & BSD & 0.1755 & 0.1955 & 0.1965 & 0.2400 & 0.2405 & 0.3255 \\
\hline & & \multirow[t]{4}{*}{150} & Nonpar & 0.2875 & 0.3165 & 0.3025 & 0.3225 & 0.3610 & 0.3665 \\
\hline & & & BNP & 0.2785 & 0.3090 & 0.2945 & 0.3140 & 0.3455 & 0.3585 \\
\hline & & & SD & 0.2755 & 0.3185 & 0.3245 & 0.3550 & 0.4305 & 0.4740 \\
\hline & & & BSD & 0.2745 & 0.3190 & 0.3245 & 0.3545 & 0.4255 & 0.4625 \\
\hline & & \multirow[t]{4}{*}{200} & Nonpar & 0.3470 & 0.3475 & 0.3935 & 0.4350 & 0.5010 & 0.6045 \\
\hline & & & BNP & 0.3440 & 0.3485 & 0.3910 & 0.4335 & 0.4925 & 0.5945 \\
\hline & & & SD & 0.3450 & 0.3365 & 0.3675 & 0.3835 & 0.4165 & 0.4660 \\
\hline & & & BSD & 0.3365 & 0.3320 & 0.3550 & 0.3720 & 0.4110 & 0.4475 \\
\hline & & \multirow[t]{4}{*}{400} & Nonpar & 0.5575 & 0.5835 & 0.6125 & 0.6540 & 0.7530 & 0.8480 \\
\hline & & & BNP & 0.5565 & 0.5845 & 0.6170 & 0.6525 & 0.7465 & 0.8375 \\
\hline & & & SD & 0.5655 & 0.5620 & 0.5690 & 0.5965 & 0.6480 & 0.7130 \\
\hline & & & BSD & 0.5645 & 0.5530 & 0.5630 & 0.5890 & 0.6395 & 0.7010 \\
\hline & \multirow[t]{12}{*}{0.9} & \multirow[t]{4}{*}{70} & Nonpar & 0.4915 & 0.5055 & 0.5155 & 0.5500 & 0.5610 & 0.6095 \\
\hline & & & BNP & 0.4515 & 0.4690 & 0.4770 & 0.5095 & 0.5140 & 0.5420 \\
\hline & & & SD & 0.4505 & 0.4770 & 0.5005 & 0.5385 & 0.5830 & 0.6475 \\
\hline & & & BSD & 0.4225 & 0.4510 & 0.4735 & 0.5120 & 0.5465 & 0.6045 \\
\hline & & \multirow[t]{4}{*}{150} & Nonpar & 0.7500 & 0.7740 & 0.7935 & 0.8035 & 0.8340 & 0.8800 \\
\hline & & & BNP & 0.7230 & 0.7400 & 0.7735 & 0.7840 & 0.8115 & 0.8550 \\
\hline & & & SD & 0.7050 & 0.7510 & 0.7785 & 0.8100 & 0.8450 & 0.9090 \\
\hline & & & BSD & 0.6900 & 0.7340 & 0.7630 & 0.8000 & 0.8335 & 0.8930 \\
\hline & & \multirow[t]{4}{*}{200} & Nonpar & 0.8255 & 0.8490 & 0.8705 & 0.8910 & 0.9245 & 0.9590 \\
\hline & & & BNP & 0.8170 & 0.8410 & 0.8615 & 0.8795 & 0.9175 & 0.9530 \\
\hline & & & SD & 0.8570 & 0.8565 & 0.8740 & 0.8790 & 0.9175 & 0.9385 \\
\hline & & & BSD & 0.8440 & 0.8380 & 0.8615 & 0.8665 & 0.9035 & 0.9260 \\
\hline
\end{tabular}


Table II. Continued.

\begin{tabular}{|c|c|c|c|c|c|c|c|c|c|}
\hline \multirow[b]{2}{*}{ Hypothesis } & \multirow[b]{2}{*}{$\rho$} & \multirow[b]{2}{*}{$N$} & \multicolumn{7}{|c|}{ ROC curve area for the standard diagnostic test } \\
\hline & & & Method & 0.60 & 0.65 & 0.70 & 0.75 & 0.80 & 0.85 \\
\hline & & 400 & Nonpar & 0.9795 & 0.9850 & 0.9890 & 0.9930 & 0.9985 & 0.9980 \\
\hline & & & BNP & 0.9770 & 0.9840 & 0.9895 & 0.9925 & 0.9985 & 0.9980 \\
\hline & & & SD & 0.9885 & 0.9920 & 0.9895 & 0.9915 & 0.9965 & 0.9960 \\
\hline & & & BSD & 0.9860 & 0.9890 & 0.9880 & 0.9915 & 0.9960 & 0.9950 \\
\hline
\end{tabular}

Note: Common correlation coefficient of the measurements between the new and standard diagnostic test. Nonpar: Non-parametric method. BNP: Bootstrap procedure of the non-parametric method. SD: Standard difference approach. BSD: Bootstrap procedure of the standard difference approach.

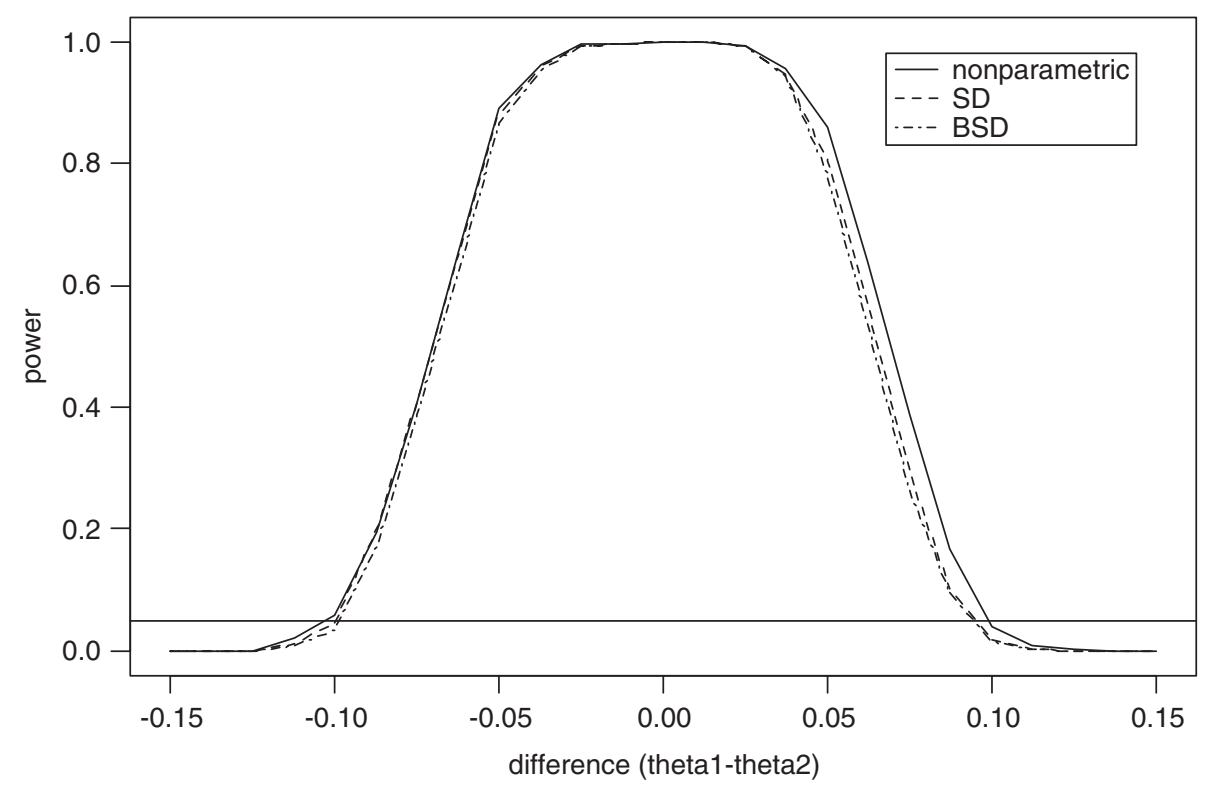

Figure 1. The empirical power curve of equivalence testing under normal distribution when the ROC curve area of the standard diagnostic test is $0.7, N=200$ and $\rho=0.9$.

difference approach can control the size at the 5 per cent nominal level and is robust to skewed distributions.

Table IV provides the empirical sizes at $\delta=0.1$ and empirical powers at $\theta_{1}-\theta_{2}=0.05$ for the ordinal data with 5 categories when the total sample size is 200 . From Table IV, the empirical sizes of the asymptotic non-parametric method and its bootstrap version for both equivalence and non-inferiority hypotheses range from 0.0760 to 0.1155 . On the other hand, the empirical sizes of the asymptotic method and bootstrap procedure of the standardized difference approach range from 0.0650 to 0.1115 for both equivalence and non-inferiority hypotheses. Although the empirical sizes of the standardized difference approach are smaller than those of the nonparametric method, the empirical sizes of all four methods for both two-sided equivalence and 


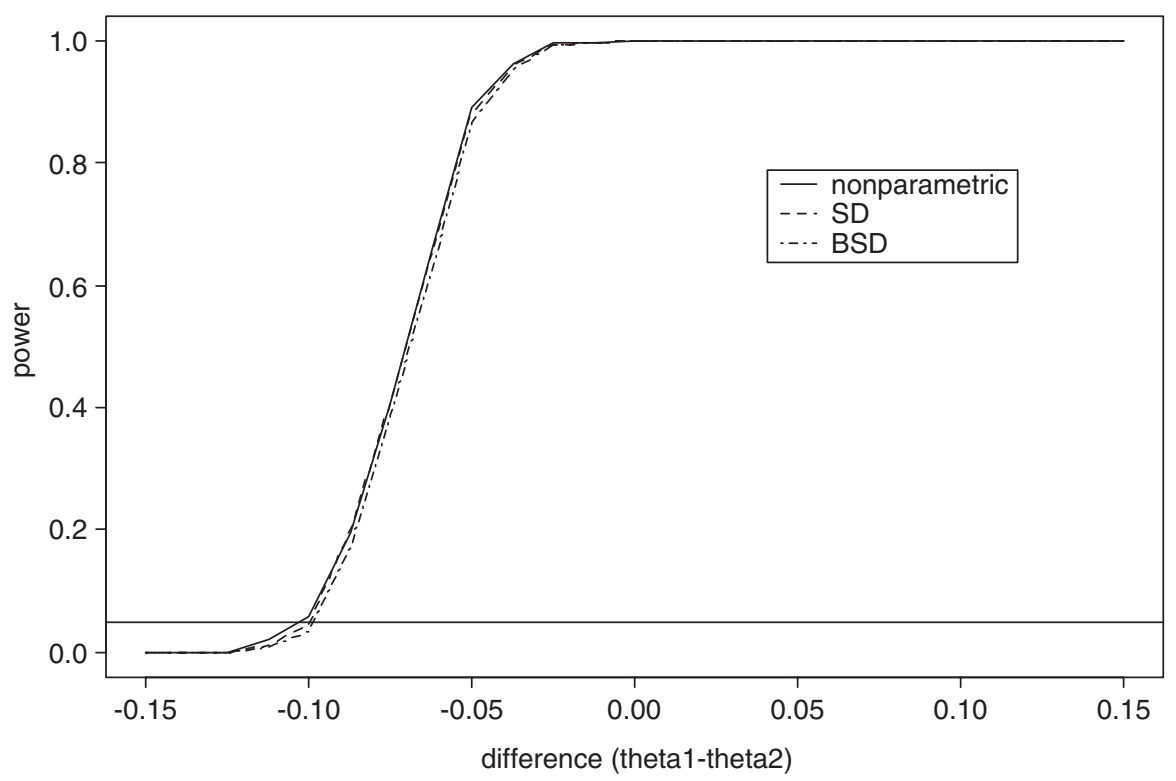

Figure 2. The empirical power curve of non-inferiority testing under normal distribution when the ROC curve area of the standard diagnostic test is $0.7, N=200$ and $\rho=0.9$.

one-sided non-inferiority hypotheses are all above 0.05487 . Therefore, the simulation results indicate that for the ordinal data, no method investigated in the simulation study can control the size at the 5 per cent nominal level.

\section{NUMERICAL EXAMPLE}

In a study by Masaryk et al. [21], two radiologists used three-dimensional magnetic resonance angiography (MRA) to evaluate the degree of arterial atherosclerotic stenosis of 65 carotid arteries (left and right) in 36 patients. These patients also underwent intra-arterial digital subtraction angiography (DSA), which is considered the gold standard for characterizing the degree of stenosis. The goals of the study were to estimate the accuracy of MRA for each reader using the area under ROC curve as the index of diagnostic accuracy, and to compare the accuracy of the two radiologists. This data set was used by Obuchowski [22] to illustrate the analysis of clustered ROC curve data. The past records showed that the average ROC curve area of experienced readers is 0.98 . Therefore, we use this data set solely for the purpose of illustration of the proposed methods for non-inferiority hypotheses on diagnostic accuracy between two readers based on the ROC curve areas. The paired measurements of 33 patients obtained from left carotid arteries were used in the example. Here, reader 2 is considered to be the experienced reader and served as the so-called 'active control' and reader 1 is the newly trained reader. Therefore, we want to verify whether the diagnostic accuracy of the new reader is not worse than that of the experienced reader. Because an average ROC curve area of 0.98 is quite high, a non-inferiority margin of -0.05 in difference of the ROC 
Table III. Empirical sizes and powers of equivalence and non-inferiority testing under the exponential distribution based on the ROC curve area for $N=200$.

\begin{tabular}{|c|c|c|c|c|c|c|}
\hline \multirow[b]{2}{*}{ Hypothesis } & \multirow[b]{2}{*}{$\rho$} & \multirow[b]{2}{*}{ Method } & \multicolumn{2}{|c|}{ Size at $\delta=0.1$} & \multicolumn{2}{|c|}{ Power at difference of 0.05} \\
\hline & & & 0.7 & 0.8 & 0.7 & 0.8 \\
\hline \multirow[t]{8}{*}{ Equivalence } & 0.5 & Nonpar & 0.1165 & 0.2795 & 0.4560 & 0.6645 \\
\hline & & BNP & 0.1145 & 0.2710 & 0.4490 & 0.6595 \\
\hline & & SD & 0.0750 & 0.0925 & 0.3615 & 0.4310 \\
\hline & & BSD & 0.0410 & 0.0370 & 0.2775 & 0.3030 \\
\hline & 0.9 & Nonpar & 0.2095 & 0.5090 & 0.8685 & 0.9730 \\
\hline & & BNP & 0.1965 & 0.4815 & 0.8595 & 0.9690 \\
\hline & & SD & 0.1030 & 0.1270 & 0.8180 & 0.8495 \\
\hline & & BSD & 0.0455 & 0.0395 & 0.7385 & 0.7330 \\
\hline \multirow[t]{8}{*}{ Non-inferiority } & 0.5 & Nonpar & 0.1165 & 0.2795 & 0.4660 & 0.6680 \\
\hline & & BNP & 0.1145 & 0.2710 & 0.4595 & 0.6625 \\
\hline & & SD & 0.0750 & 0.0925 & 0.3800 & 0.4405 \\
\hline & & BSD & 0.0410 & 0.0370 & 0.3015 & 0.3215 \\
\hline & 0.9 & Nonpar & 0.2095 & 0.5090 & 0.8685 & 0.9730 \\
\hline & & BNP & 0.1965 & 0.4815 & 0.8595 & 0.9690 \\
\hline & & SD & 0.1030 & 0.1270 & 0.8180 & 0.8495 \\
\hline & & BSD & 0.0455 & 0.0395 & 0.7385 & 0.7330 \\
\hline
\end{tabular}

Note: $\rho$ : Common correlation coefficient of the measurements between the new and standard diagnostic test. Nonpar: Non-parametric method. BNP: Bootstrap procedure of the non-parametric method. SD: Standard difference approach. BSD: Bootstrap procedure of the standard difference approach.

curve area is considered in the example. Under normal assumption and a non-inferiority margin of -0.05 based on the ROC curve area, because $\Phi^{-1}(0.98)=2.05375$ and $\Phi^{-1}(0.93)$ is 1.47579 , the corresponding non-inferiority margin for the standardized difference approach is $1.48579-2.05375=-0.57796$.

The non-parametric estimates of the ROC curve area are 0.988 for reader 1 and is 0.984 for reader 2. The standard deviation of $\hat{\theta}_{1}-\hat{\theta}_{2}$ is estimated to be 0.0056 . It follows that $Z_{l}=9.64$, which is greater than $Z_{0.05}=1.645$. Therefore, as compared to reader 2, the non-inferiority of reader 1 with respect to diagnostic accuracy based on the ROC curve area can be concluded at the 5 per cent significance level with respect to the non-inferiority limit of -0.05 . The 95 per cent lower bootstrap confidence limit for the difference in the ROC curve areas based on the non-parametric method is 0 which is greater than -0.05 . Therefore, the same conclusion for the non-inferiority hypothesis is also reached at the 5 per cent significance level.

On the other hand, under normal assumption, $\hat{\lambda}_{1}=1.86948$ and $\hat{\lambda}_{2}=1.63863$. The estimated standard deviation of $\hat{\lambda}_{1}-\hat{\lambda}_{2}$ is 0.2452 . Therefore, for $\varepsilon_{L}=-0.57796\left(\delta_{L}=-0.05\right), Z_{L}^{\prime}=3.30$ that is greater than $Z_{0.05}=1.645$ too. As compared to reader 2 , the non-inferiority of reader 1 can be concluded at the 5 per cent significance level with the non-inferiority margin of -0.57796 . The 95 per cent lower bootstrap confidence interval for $\lambda_{1}-\lambda_{2}$ based on the standardized difference is -0.080610 , which is greater than the non-inferiority margin of -0.57796 . It follows that the same conclusion for non-inferiority hypothesis is also reached at the 5 per cent significance level by the bootstrap version of the standardized difference approach. 
Table IV. Empirical sizes and powers of equivalence and non-inferiority testing under the ordinal data based on the ROC curve area for $N=200$.

\begin{tabular}{|c|c|c|c|c|c|c|}
\hline \multirow[b]{3}{*}{ Hypothesis } & \multirow[b]{3}{*}{$\rho$} & \multirow[b]{3}{*}{ Method } & \multicolumn{4}{|c|}{ ROC curve area for the standard diagnostic test } \\
\hline & & & \multicolumn{2}{|c|}{ Size at $\delta=0.1$} & \multicolumn{2}{|c|}{ Power at difference of 0.05} \\
\hline & & & 0.7 & 0.8 & 0.7 & 0.8 \\
\hline \multirow[t]{8}{*}{ Equivalence } & \multirow[t]{4}{*}{0.5} & Nonpar & 0.0875 & 0.0775 & 0.4380 & 0.5430 \\
\hline & & BNP & 0.0885 & 0.0760 & 0.4360 & 0.5405 \\
\hline & & SD & 0.0720 & 0.0765 & 0.3690 & 0.4435 \\
\hline & & BSD & 0.0670 & 0.0650 & 0.3440 & 0.4150 \\
\hline & \multirow[t]{4}{*}{0.9} & Nonpar & 0.1155 & 0.1125 & 0.8115 & 0.8805 \\
\hline & & BNP & 0.1115 & 0.1090 & 0.8055 & 0.8765 \\
\hline & & SD & 0.0830 & 0.1115 & 0.7110 & 0.7890 \\
\hline & & BSD & 0.0730 & 0.0925 & 0.6895 & 0.7685 \\
\hline \multirow[t]{8}{*}{ Non-inferiority } & \multirow[t]{4}{*}{0.5} & Nonpar & 0.0875 & 0.0775 & 0.4480 & 0.5445 \\
\hline & & BNP & 0.0885 & 0.0760 & 0.4465 & 0.5420 \\
\hline & & SD & 0.0720 & 0.0765 & 0.3820 & 0.4455 \\
\hline & & BSD & 0.0670 & 0.0650 & 0.3580 & 0.4180 \\
\hline & \multirow[t]{4}{*}{0.9} & Nonpar & 0.1155 & 0.1125 & 0.8115 & 0.8805 \\
\hline & & BNP & 0.1115 & 0.1090 & 0.8055 & 0.8765 \\
\hline & & SD & 0.0830 & 0.1115 & 0.7110 & 0.7890 \\
\hline & & BSD & 0.0730 & 0.0925 & 0.6895 & 0.7685 \\
\hline
\end{tabular}

Note: Common correlation coefficient of the measurements between the new and standard diagnostic test. Nonpar: Non-parametric method. BNP: Bootstrap procedure of the non-parametric method. SD: Standard difference approach. BSD: Bootstrap procedure of the standard difference approach.

\section{DISCUSSION}

The technology of diagnostic tests for disease identification and staging advanced rapidly. In particular, after completion of the Human Genome Project, tests based on gene chips or biochips may provide quick, inexpensive, non-invasive and easy-to-use tools for diagnosis of diseases. Furthermore, importance of diagnostic tests increases as more targeted clinical trials will be conducted for the individualized treatment of patients in the genomic era [23,24]. However, the diagnostic accuracy of any newly developed diagnostic technology must be rigorously evaluated and approved by the health regulatory agencies before their routine use. One approach is to verify whether the diagnostic accuracy of the new diagnostic procedure is equivalent or is not worse than that of the current standard procedure due to the other advantages offered by the new procedure. Because the ROC curve area is a measure for separation of the distribution of the measurements of the diseased patients from that of the non-diseased subjects, we proposed to use the standardized difference for evaluation of equivalence and non-inferiority between the new and standard diagnostic procedures. A FORTRAN program for computation of all four methods is from the authors upon request.

Simulation results indicate that the non-parametric method may inflate the size considerably when the underlying distribution is skewed. On the other hand, in terms of size and power, the standardized difference approach is a very competitive alternative to the non-parametric 
method. In particular, the bootstrap method of the standardized difference approach not only controls the size at the nominal level for both normal and exponential distributions but also provides sufficient power. Therefore, simulation results suggested that the bootstrap method of the standardized difference approach is quite robust to the skewedness of the distribution and selection of the equivalence limits under the skewed distributions. As a result, we recommend the bootstrap method of the standardized difference approach to evaluate the two-sided equivalence and one-sided non-inferiority hypotheses based on the paired areas under the ROC curves between the new and standard diagnostic procedures when the measurements of both procedures are continuous.

However, all four methods, including the non-parametric method, fail to adequately control the size for the ordinal data. One of the possible reasons for the poor performance of the four methods with respect to ordinal data is that the variance of the ordinal (categorical) data is a function of the mean. The restricted maximum likelihood estimator (RMLE) of the variance of categorical data obtained at the equivalence limit should be used for testing the equivalence or non-inferiority hypotheses. Wald-type asymptotic methods for evaluation of equivalence or non-inferiority for categorical data generally will inflate the size [2]. As a result, extreme caution should be taken when to evaluate the equivalence and non-inferiority based on the ROC curve areas computed from the ordinal data. Further research on assessment of equivalence for the ordinal data is warranted since they are the most commonly recorded form of data in diagnostic procedures.

Equivalence limits should be determined jointly by clinicians, radiologists, and statisticians and pre-specified in the study protocol before the conduct of the study. Furthermore, determination of equivalence limits is not an easy task and many factors such as the usage of diagnostic tests and the accuracy of the standard diagnostic test, feasibility of the required sample size and many others should be considered. Because any equivalence or non-inferiority diagnostic study includes the standard diagnostic test, it is also an active control equivalence study. Therefore, the issues of assay sensitivity, constancy assumption, and reasons for selection of equivalence limits should be adequately addressed in the protocol [25]. Population bioequivalence and individual bioequivalence [26-29] have been suggested to evaluate bioequivalence for approval of generic drugs and to assess equivalence between diagnostic technologies [30,31]. However, both population and individual bioequivalence are based on aggregate criteria of population average, intra-subject variability and variance of the subject $\times$ formulation interaction. It turns out that two totally different distributions with different averages and variances can be concluded individual bioequivalent [32]. Because of this drawback and other issues, the US FDA [33] and other health regulatory agencies in the world still employ the average bioequivalence as the criterion for approval of generic drugs. Therefore, we also focus on average accuracy for assessment of equivalence between diagnostic tests. However, equivalence or non-inferiority on variability between diagnostic procedures is equally important and requires further research.

\section{ACKNOWLEDGEMENTS}

We like to thank the two anonymous reviewers for their careful, thoughtful and thorough review and comments which greatly improved the content and presentation of our work. This work is partially supported by the Taiwan National Science Grants: NSC 92-2118-M-006-001 and NSC 93-2118-M006-002. 


\section{REFERENCES}

1. Schuirmann DJ. A comparison of the two one-sided tests procedure and the power approach for assessing the equivalence of bioavailability. Journal of Pharmacokinetics and Biopharmaceutics 1987; 15:657-680.

2. Liu JP, Hsueh HM, Hsieh E, Chen JJ. Tests for equivalence or non-inferiority for paired binary data. Statistics in Medicine 2002; 21:231-245.

3. Hsueh HM, Liu JP, Chen JJ. Unconditional exact tests for equivalence or non-inferiority for paired binary endpoints. Biometrics 2001; 57:478-483.

4. Tang NS, Tang ML, Chan ISF. On tests of equivalence via non-unity relative risk for matched-pair design. Statistics in Medicine 2003; 22:1217-1233.

5. Metz CE. Basic principles of ROC analysis. Seminars in Nuclear Medicine 1978; VIII:283-298.

6. Hanley JA, McNeil BJ. The meaning and use of the area under a receiver operating characteristic (ROC) curve. Radiology 1982; 143:29-36.

7. Obuchowski N. Testing for equivalence of diagnostic tests. American Journal of Radiology 1997; 168:13-17.

8. Zhou XH, Obuchowski NA, McClish DK. Statistical Methods in Diagnostic Medicine. Wiley: New York, 2002; 188-192.

9. DeLong E, DeLong D, Clarke-Pearson D. Comparing the areas under two or more correlated receiver operation characteristic cures: a non-parametric approach. Biometrics 1988; 44:837-845.

10. Sen PK. On some convergence properties of $U$-statistics. Calcutta Statistical Association Bulletin 1960; 10: $1-18$.

11. Berger RL. Multiparameter hypothesis testing in acceptance testing. Technometrics 1982; 24:295-300.

12. Hauck WW, Hyslop T, Anderson S. Generalized treatment effects for clinical trials. Statistics in Medicine 19:887-899.

13. Reiser B, Guttman I. Statistical inference for $P(Y<X)$ : the normal case. Technometrics 1986; 28:253-257.

14. Serfling RJ. Approximation Theorems of Mathematical Statistics. Wiley: New York, 1978; 19-21.

15. Efron B, Tibshirani RJ. Bootstrap methods for standard errors, confidence intervals, and other measures of statistical accuracy. Statistical Science 1986; 1:54-77.

16. Efron B, Tibshirani RJ. An Introduction to the Bootstrap. Chapman \& Hall: New York, 1993; $168-176$.

17. Moran PAP. Testing for correlation between non-negative variates. Biometrika 1967; 54:385-394.

18. Obuchowski NA. Receiver operating characteristic curves and their use in radiology. Radiology 2003; 229: $3-8$.

19. Parker CB, Delong ER. ROC methodology within a monitoring framework. Statistics in Medicine 2003; 22: $3473-3488$.

20. Pepe MS. The Statistical Evaluation of Medical Tests for Classification and Prediction. Oxford University Press: Oxford, U.K., 2003; 96-127.

21. Masaryk AM, Ross JS, DiCello MC, Modic MT, Paranandi L, Masaryk TJ. 3DFT MR angiography of the carotid bifurcation: potential and limitations as a screening examination. Radiology 1991; 179:797-804.

22. Obuchowski N. Nonparametric analysis of clustered ROC curve data. Biometrics 1997; 53:567-578.

23. Simon R, Maitournan A. Evaluating the efficiency of targeted designs for randomized clinical trials. Clinical Cancer Research 2004; 10:6759-6763.

24. Maitournan A, Simon R. On the efficiency of targeted clinical trials. Statistics in Medicine 2005; 24:329-339.

25. Chow SC, Liu JP. Design and Analysis of Clinical Trials (2nd edn). Wiley: New York, 2004; $250-265$.

26. Anderson S, Hauck WW. Consideration of individual bioequivalence. Journal of Pharmacokinetics and Biopharmaceutics 1990; 18:259-273.

27. Hauck WW, Anderson S. Types of bioequivalence and related statistical considerations. International Journal of Clinical Pharmacology, Therapeutics and Toxicology 1992; 30:181-187.

28. Chen ML. Individual bioequivalence-a regulatory update. Journal of Biopharmaceutical Statistics 1997; 7 $5-11$.

29. US FDA Guidance for Industry on Statistical Approaches to Establishing Bioequivalence. CDER, FDA: Rockville, MD, 2001; 3-7.

30. Obuchowski N. Film-screen versus digitized mammography: assessment of clinical equivalence. American Journal of Radiology 1999; 173:889-894.

31. Obuchowski N. Can electronic images replace hard-copy film? Defining and testing the equivalence of diagnostic tests. Statistics in Medicine 2001; 20:2845-2863.

32. Liu JP. Statistical evaluation of individual bioequivalence. Communications in Statistics, Theory and Methods 1998; 27:1433-1451.

33. US FDA Guidance for Industry on Bioavailability and Bioequivalence Studies for Orally Administered Drug Products-General Considerations. CDER, FDA: Rockville, MD, 2003; 11. 